\title{
FLEBOTOMÍNEOS (DÍPTERA: PSYCHODIDAE) DE UMA FLORESTA PRIMÁRIA DE TERRA FIRME DA ESTAÇÃO EXPERIMENTAL DE SILVICULTURA TROPICAL, ESTADO DO AMAZONAS, BRASIL.
}

\author{
Artur Gomes DIAS-LIMA ${ }^{1}$, Eloy G. CASTÉLLON²; Ítalo SHERLOCK ${ }^{1}$
}

\begin{abstract}
RESUMO - Estudos sobre o levantamento da fauna de flebótomos foram realizados numa floresta primária de terra firme da Estação Experimental de Silvicultura Tropical (EEST) do Instituto Nacional de Pesquisas da Amazônia (INPA), durante os meses de outubro de 1998 a março de 1999. Utilizando-se de armadilhas luminosas CDC colocadas a $1 \mathrm{~m}, 10 \mathrm{~m}$ e 20 metros de altura do solo, foram coletados 7.409 flebótomos, pertencentes a dois gêneros, Lutzomyia $(99,98 \%$ ) e Brumptomyia (0,02\%), abrangendo 39 espécies. Dentro do gênero Lutzomyia, os subgêneros mais representados foram Nyssomyia, com 39,43\%, e Psychodopygus com 22,68\%. O número de flebotomíneos coletados foi crescente, a partir do início da estação chuvosa.
\end{abstract}

Palavras-chave: flebotomíneos, leishmaniose, Amazônia.

\section{Sandflies (Diptera: Psychodidae) in a primary non-flooded forest of Experimental Station of Tropical Forestation, Amazonas State, Brazil.}

\begin{abstract}
Studies about sand flies fauna were carried out in a non-flooded primary forest at the Experimental Station of Tropical Forest - National Institute of Amazon Research, between October 1998 and March 1999. CDC light traps were placed at 1m, 10m and 20 meters above ground level. 7.409 phlebotomines were collected, belonging to the genera, Lutzomyia $(99,98 \%)$ and Brumptomyia (0,02\%), represented by 39 species. In the genus Lutzomyia, the more frequent subgenus was Nyssomyia, with 39,43\%, followed by Psychodopygus with 22,68\%. The number of sandflies collected was increased, from the beginning of the rainy station.
\end{abstract}

Key-words: sandflies, leishmaniasis, Amazon.

\section{INTRODUÇÃO}

A continuidade dos levantamentos padronizados de flebótomos nas diversas regiões e ecossistemas da Amazônia é importante sob vários aspectos, não só para a entomologia médica, como por exemplo, a descoberta de novas leishmanias neotropicais, como também indicar áreas de conservação biológica. Além disso, a análise da diversidade das faunas de flebótomos pode indicar não somente regiões ecologicamente distintas, como também servir como base para detectar futuras mudanças a locais sujeitos a impactos ambientais, identificando áreas para conservação biológica (Barrett et al., 1991; 1996).

A região Amazônica apresenta uma alta riqueza de espécies de flebótomos e altos índices de diversidade de ecótopos naturais na floresta de terra firme. Apesar disso, os estudos

${ }^{1}$ Laboratório de Parasitologia / Entomologia, Centro de Pesquisas Gonçalo Moniz, Fundação Oswaldo Cruz. Rua Waldemar Falcão, 121, Brotas, Salvador, BA, 40295-001, Brasil.

${ }^{2}$ Coordenação de Pesquisas em Ciências da Saúde, Instituto Nacional de Pesquisas da Amazônia. Av. André Araújo, 2936, Petrópolis, Manaus, AM 69011-970, Brasil.

Fontes financiadoras: Coordenação do Curso de Pós-graduação - CCPG do Instituto Nacional de Pesquisas da Amazônia - INPA e Coordenação de Aperfeiçoamento de Pessoal de Nível Superior - CAPES. 
com enfoque ecológico das espécies de flebótomos amazônicas, são limitadas aos vetores de leishmanias, e mesmo para estes, são relativamente raros (Pessoa, 2000).

Segundo Lainson \& Shaw (1979) o conhecimento das flutuações das populações dos flebótomos, permite orientar para que se evite freqüentar os seus habitats, principalmente durante os períodos do ano em que são mais abundantes, possibilitando assim o controle da transmissão para o homem.

Para a aplicação de medidas corretas de controle da endemia é imperativa a realização de observações sobre a bionomia e a ecologia das espécies de flebótomos, tais como a variação na atividade estacional e horária, correlacionando-as com dados climáticos e com as fases lunares, a variação da distribuição horizontal e vertical, antropofilia e infecção natural e experimental por leishmanias (Sherlock et al., 1996).

Estudos detalhados da dinâmica de transmissão, fatores de risco em populações humanas expostas, correlação de variáveis bióticas e abióticas com incidência e prevalência de infecção e a evolução de efeitos de alterações ambientais, são extremamente necessárias para a adoção de medidas práticas a fim de reduzir a exposição do homem aos vetores (Barrett, 1993).

Apesar do número considerável de espécies de flebótomos no Continente americano, apenas uma pequena percentagem dessas são suspeitas e/ou comprovadas vetoras de leishmanioses. Existem muitas evidências que algumas espécies de leishmanias podem ser transmitidas somente por certas espécies de flebótomos, pela íntima associação entre lipofosfoglicanos (LPGs) do parasito e receptores de membrana dos vetores (Pimenta et al., 1994).

A Leishmaniose Tegumentar Americana (LTA) no Município de Manaus, Estado do Amazonas, tem acompanhado as ocupações populacionais na periferia da cidade e ao longo de duas estradas (BR 174 Manaus - Presidente Figueiredo e AM 010 Manaus-Itaquatiara) onde tem ocorrido freqüentes assentamentos de projetos agrícolas e habitacionais, além de outras atividades humanas. A maior prevalência da doença acontece no período de novembro à abril, período com maiores índices pluviométricos na região, e quando há aumento na população e atividade dos vetores transmissores (Guerra et al., 2001).

O objetivo principal deste trabalho foi realizar um levantamento da fauna de flebó tomos vetores de leishmanias, em uma floresta primária de terra firme preservada de uma área endêmica de LTA e acompanhar a freqüência de distribuição mensal desses dípteros durante a estação chuvosa, período de maior incidência de casos de leishmanioses.

\section{MATERIAL E MÉTODOS}

O trabalho foi realizado na Estação Experimental de Silvicultura Tropical (EEST) do Instituto Nacional de Pesquisas da Amazônia - INPA, localizada ao norte de Manaus, no Km 45 da Rodovia BR 174 que liga Manaus à Boa Vista, entre as coordenadas geográficas $2^{\circ} 47^{\prime}$ 05"S; 60 11' 51" W (Figura 1).

Este local foi escolhido por ser uma área endêmica para LTA (Figuras 2 e 3 ) com inúmeros casos registrados anualmente na Fundação de Medicina Tropical do Amazonas - FMTAM (dados não publicados). Além disso, outros critérios utilizados foram a localização próximo à Manaus, pela sua composição vegetal, com presença de floresta de terra firme preservada, pela sua composição faunística e pela epidemiologia da doença ser pouco conhecida ao longo da rodovia BR 174.

O clima da região, segundo a classificação de Köppen, é do tipo Am, que caracteriza a zona tropical úmida. A temperatura média anual é de $27,6^{\circ} \mathrm{C}$, a precipitação anual é de $2478 \mathrm{~mm}$ e a umidade relativa do ar é de 85 a 95\%. A estação seca ocorre entre junho e novembro. Predomina na região o latossolo amarelo textura pesada a muito pesada, com sedimentos da formação Alter do Chão do Cretáceo superior, consistindo de arenitos caulínicos, argilitos, grauvacas e brechas intraformacionais. O relevo é ondulado com presença de platôs com extensão de 500 à 1000 metros (Freitas \& Higuchi, 1993).

A área é coberta por floresta tropical úmida densa de terra firme, típica da parte central da região amazônica. $\mathrm{O}$ estrato superior desta floresta é composto de árvores que 
variam de tamanho e que alcançam até 40 metros de altura, onde predominam três famílias botânicas: Lecythidaceae, Leguminoceae e Sapotaceae. Dentre as árvores, presentes na área de estudos, de grande porte e com raízes tabulares ou troncos com cascas, destacam-se: "Angelim-pedra" Dinizia excelsa (Ducke), Maçaranduba Manilkara huberi (Ducke)
Standl, e Castanha - de - macaco Cariniana micrantha Ducke.

O material biológico foi coletado no período de seis meses, de outubro (menor regime hídrico) de 1998 à março (maior regime hídrico) de 1999, em excursões mensais que duraram cinco dias (quatro noites), durante a fase de lua nova, no período indicado.

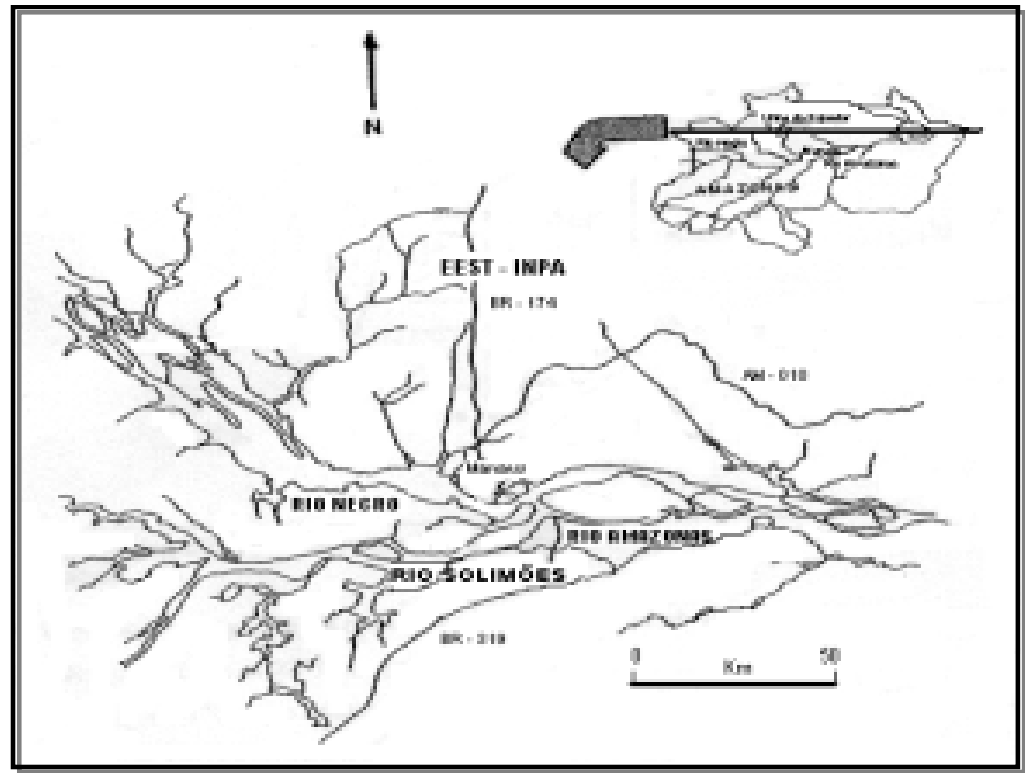

Figura 1: Mapa da Amazônia Central Brasileira, mostrando a localização da Estação de Silvicultura Tropical do Instituto Nacional de Pesquisas da Amazônia, Amazonas, Brasil.

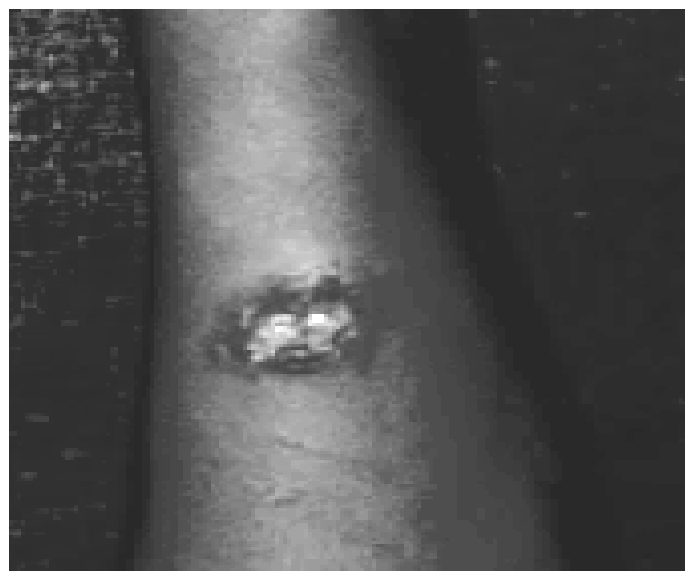

Figura 2 - Lesão de Leishmaniose Tegumentar Americana autóctone da rodovia BR-174 - AM, no braço de um paciente, registrado no Instituto de Medicina Tropical do Amazonas, no ano de 1998. 


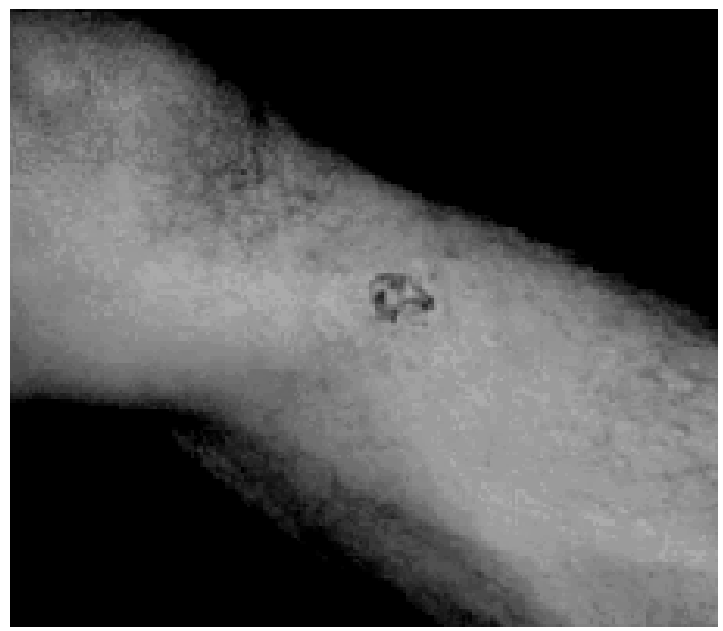

Figura 3 - Lesão de Leishmaniose Tegumentar Americana em tratamento, na perna de um funcionário da Estação Experimental de Silvicultura Tropical - INPA.

Em uma área de floresta primária de terra firme (Figura 4) de aproximadamente 1 hectare, foi realizada a coleta dos flebótomos, utilizando-se de 18 armadilhas luminosas CDC “miniatura” (Hausher's Machine Works ${ }^{\circledR}$, New Jersey, EUA), sendo colocadas seis armadilhas a 1,10 e $20 \mathrm{~m}$ de altura do solo. As armadilhas foram colocadas durante quatro noites consecutivas por mês, no crepúsculo, entre 17:00 e 6:00 horas do dia seguinte.

Inicialmente foi demarcada no platô uma trilha principal de $100 \mathrm{~m}$ de comprimento. No início e no final da trilha principal foram delineados dois transectos paralelos, no sentido SE-NO, com comprimento de $50 \mathrm{~m}$ para direita e para esquerda (trilhas A,B,C e D) (Figura 5). No final destas trilhas, ou seja, nos transectos de $50 \mathrm{~m}$ de ambos os lados e na trilha principal, foram colocadas armadilhas à $1 \mathrm{~m}, 10 \mathrm{~m}$ e $20 \mathrm{~m}$ de altura.

Todas as espécies foram identificadas de acordo com a classificação de Young \& Duncan (1994).

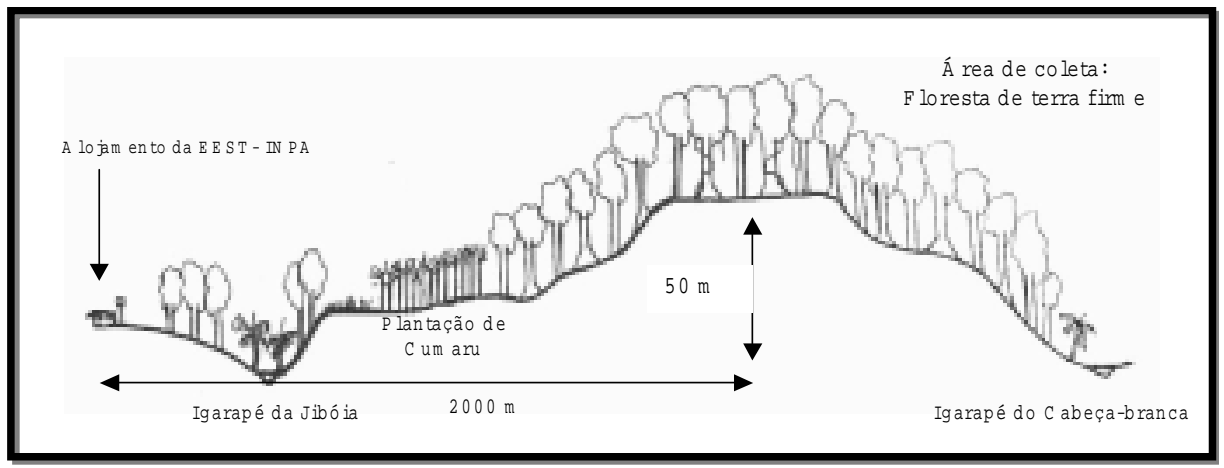

Figura 4 - Corte seccional transversal, demonstrando as características do relevo da área de estudo. 


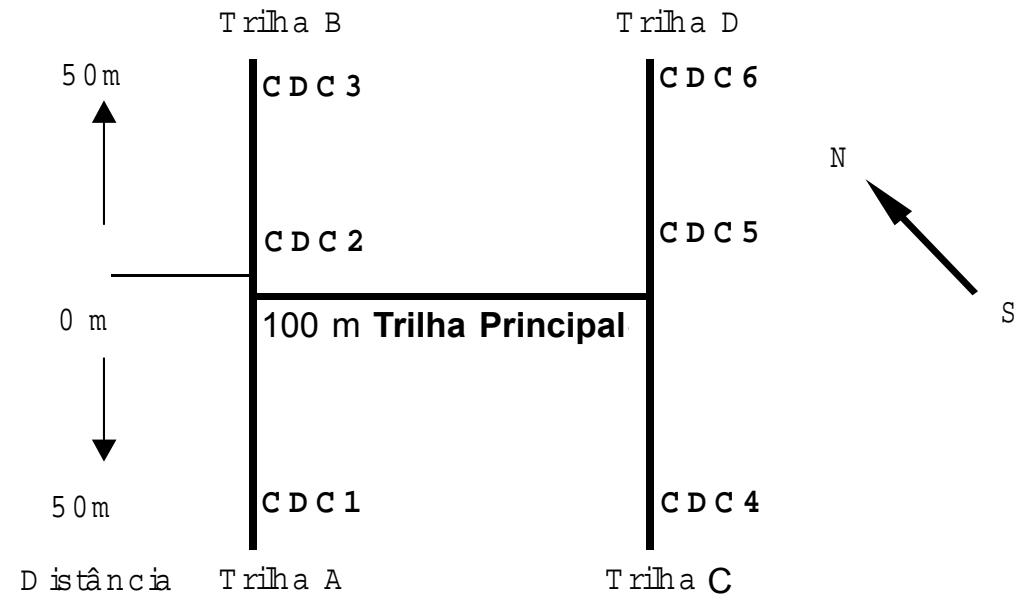

Figura 5 - Desenho experimental do local de coleta com armadilhas luminosas colocadas a 1, 10 e 20 metros de altura, demonstrando a trilha principal e as trilhas secundárias A,B,C e D (transectos) com 50m.

\section{RESULTADOS}

Foram coletados um total de 7.409 flebótomos de 39 espécies diferentes, agrupadas em dois gêneros, Brumptomyia, com 0,02\% e Lutzomyia, com 99,98\% (Tabela 1). Do gênero Lutzomyia, foram coletadas espécies pertencentes a 11 subgêneros e 6 grupos.

O maior número de flebótomos coletadas está presente no subgênero Nyssomyia, com $39,43 \%$, onde as espécies $L$. umbratilis e $L$. anduzei, contribuíram com 18,82 e $14,18 \%$ respectivamente, ou seja, juntas somaram $33 \%$ dos indivíduos coletados.

Outros subgêneros bem representados foram Psychodopygus com 22,68\% e Trichopygomyia com $10,69 \%$. A presença da espécie $L$. trichopyga, com $10,47 \%$ do total dos flebótomos coletados, favoreceu a representatividade do subgênero Trichopygomyia. Dessa mesma forma, L. rorotaensis com $7,35 \%$ foi bem representativa no grupo Oswaldoi e L. infraspinosa $(5,34 \%)$ no subgênero Evandromyia.

As espécies menos representativas foram as do gênero Brumptomyia, B. bragai e $B$. $s p$., do grupo migonei, L. pacae, do grupo verrucarum, L. sp., do grupo pilosa, L. pilosa, do subgênero Psychodopygus L. bispinosa, do subgênero Micropygomyia, L. cayennensis e finalmente, do grupo aragaoi, L. inflata.
Das 39 espécies, foram coletadas fêmeas em maior número em relação aos machos (Tabela 1 ), onde $L$. tuberculata, $L$. infraspinosa e $L$. rorotaensis, apresentaram as maiores diferenças entre machos e fêmeas coletadas. Nas espécies restantes, foi observada uma relação próxima de 1 macho para cada fêmea coletada. Quanto a espécie L. umbratilis, a relação foi de duas fêmeas para cada macho coletado.

O ciclo de chuvas na Amazônia Central brasileira está dividido em duas estações distintas, sendo uma a estação chuvosa, que se inicia no mês de novembro e termina aproximadamente no mês de abril. Quando começa a haver um declínio nos índices pluviométricos, inicia-se a estação seca, que abrange os meses de maio à outubro. Durante o período da estação chuvosa, outros fatores abióticos como temperatura $\left({ }^{\circ} \mathrm{C}\right)$ e umidade relativa do ar (\%), variam de forma peculiar, quando diminui a temperatura média e aumenta a umidade relativa, acontecendo o inverso durante a estação seca.

Quando comparamos uma frequência do número de casos acumulativos com a distribuição mensal dos flebótomos coletados em nossa área de estudos (Figura 6), podemos observar uma tendência ao aumento do número de flebótomos concomitante ao aumento do número de casos, onde ambos ocorrem no início da estação chuvosa. 


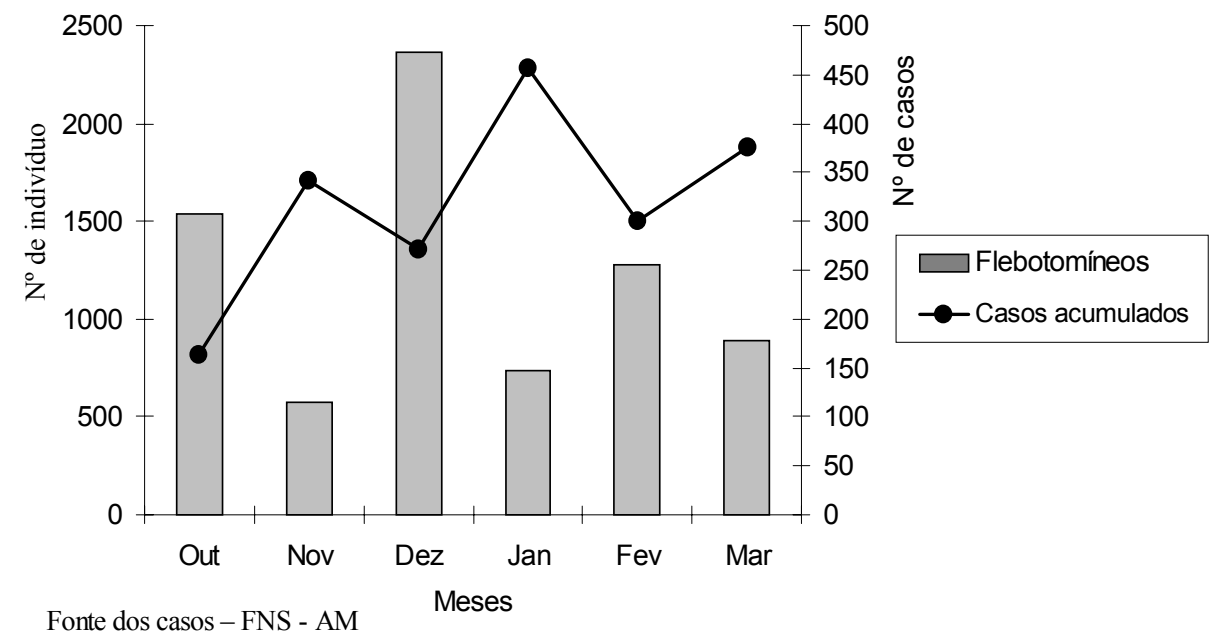

Figura 6 - Casos humanos mensais de Leishmaniose Tegumentar Americana - LTA - nos municípios de Manaus, Presidente Figueiredo (BR 174) e Itaquatiara (AM 010), durante o período de 1985 à 1995, em relação a distribuição mensal dos flebotomíneos.

Quanto a distribuição das quatro espécies do subgênero Nyssomyia (Figura 7) podemos observar uma certa similaridade no número de flebótomos coletados das espécies L. umbratilis e L. anduzei. Essas espécies foram coletadas com frequência no mês de outubro, e oscilaram nos meses subsequentes, sendo coletadas novamente com relativa abundância no mês de dezembro. A espécie $L$. olmeca nociva foi capturada com maior frequência em relação a L. flaviscutellata, apresentando um pico maior no mês de dezembro.

Dentre as espécies do subgênero Psychodopygus (Figura 8), L. davisi foi a espécie mais abundante e $L$. geniculata a menos abundante. Quanto a frequência mensal, as espécies desse subgênero, oscilaram nos meses de coleta, com picos no mês de dezembro. As espécies L. amazonensis, L. squamiventris squamiventris, L. ayrozai e L. paraensis apresentaram-se com maior frequência nos meses de outubro e dezembro, sendo este ultimo mês o de maior frequência. Quanto a espécie $L$. geniculata, apresentou-se de forma diferente das demais espécies do subgênero Psychodopygus, aumentando a frequência de indivíduos, a partir do mês de janeiro a março. Em relação a frequência mensal de outras espécies coletadas com relativa frequência (Figura 9), L. trichopyga foi a mais abundante e L. dendrophyla a menos abundante. A espécie L. trichopyga apresentou dois picos marcantes de frequência, um menor no mês de outubro e um maior no mês de dezembro, diminuindo acentuadamente os indivíduos coletados após este mês. Manteve-se estável entre os meses de janeiro a março, apesar de uma pequena quantidade de indivíduos coletados.

As espécies $L$. rorotaensis e $L$. infraspinosa apresentaram uma queda progressiva na frequência de indivíduos, a partir do mês de outubro, acentuando-se após o mês de dezembro, e mantendo-se estáveis entre os meses de fevereiro e março.

A espécie $L$. gomezi apesar de coletada em pequeno número, revelou dois pequenos picos de frequência, um no mês outubro e outro em dezembro. As espécies L.tuberculata, $L$. furcata, L. shannoni e L. dendrophyla, apesar de terem sido coletadas em pequeno número, mostraram que estão distribuídas de uma forma homogênea ao longo dos meses de outubro a março. 


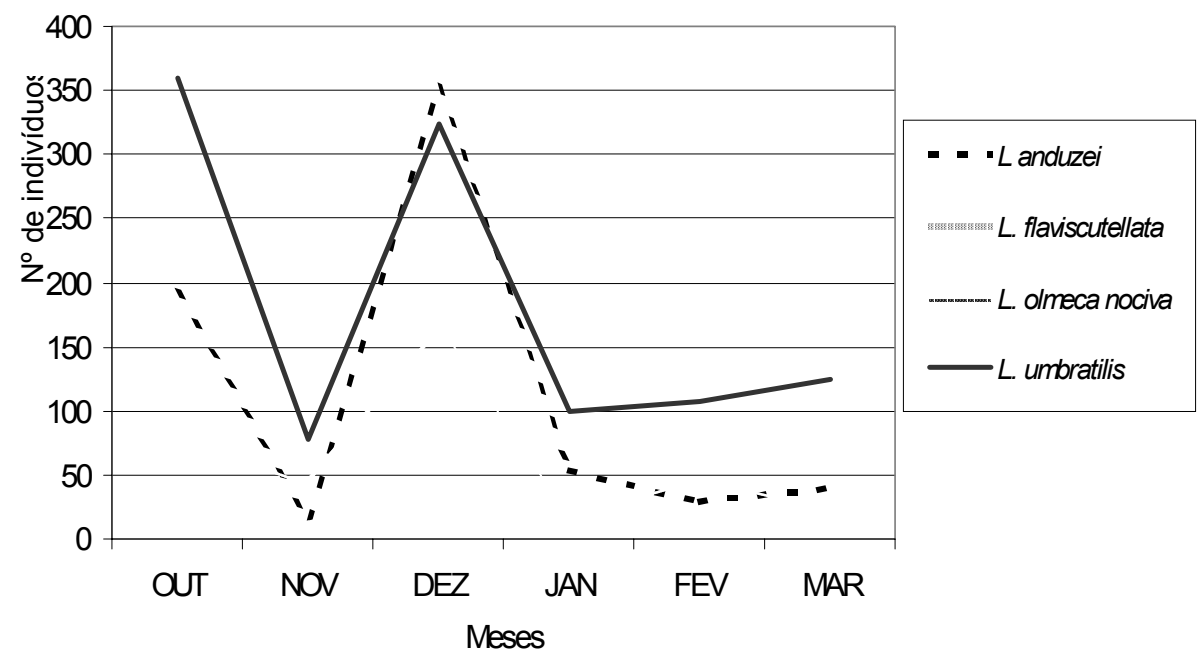

Figura 7 - Frequência mensal das espécies do sub-gênero Nyssomyia, coletadas com armadilhas luminosas CDC na Estação Experimental de Silvicultura Tropical - INPA - AM, durante o período de outubro de 1998 a março de 1999.

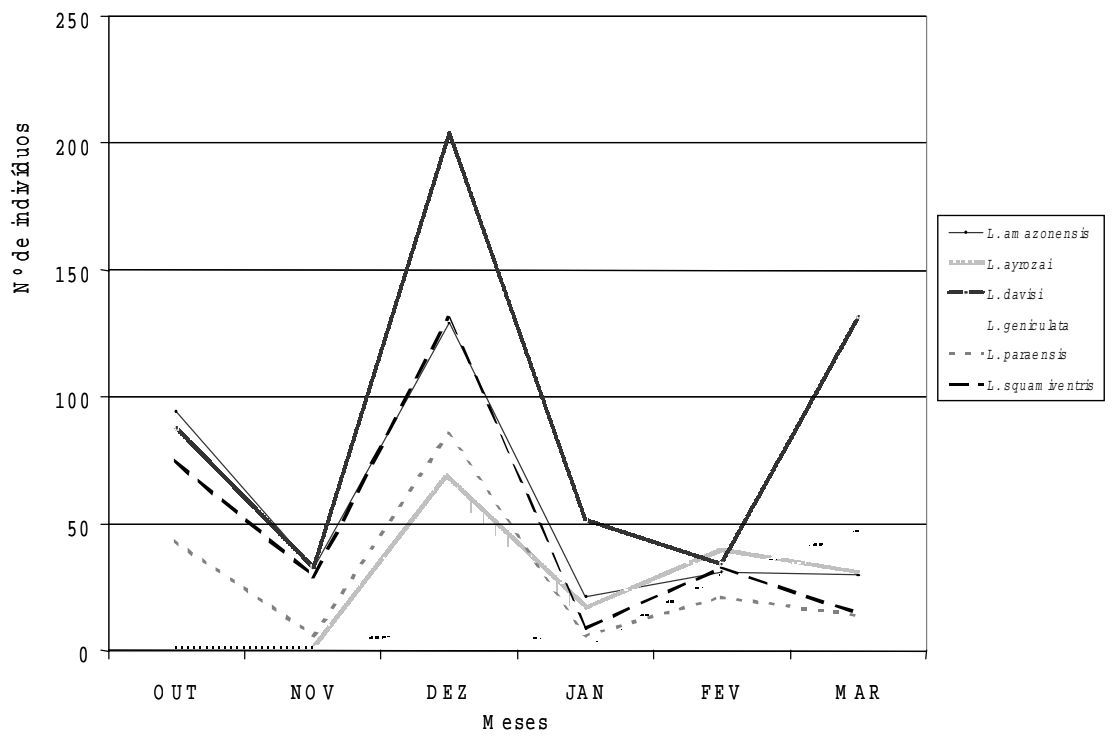

Figura 8 - Frequência mensal das espécies do sub-gênero Psychodopygus, coletadas com armadilhas luminosas CDC na Estação Experimental de Silvicultura Tropical - INPA - AM, durante o período de outubro de 1998 a março de 1999. 


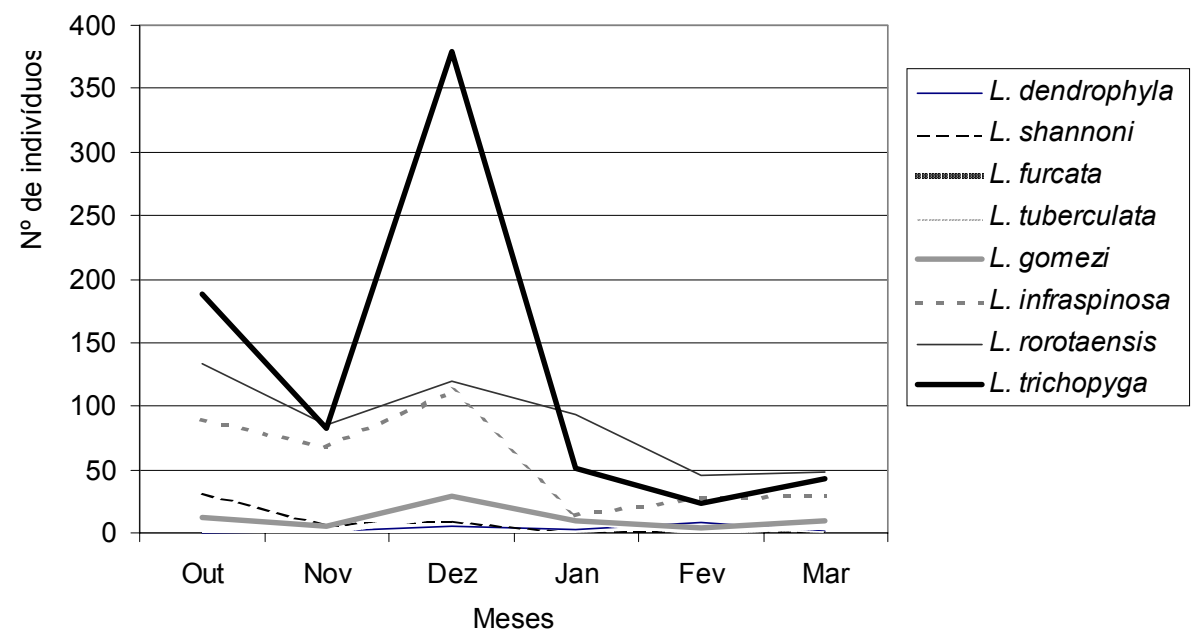

Figura 9 - Frequência mensal de espécies de flebotomíneos, coletadas com armadilhas luminosas CDC na Estação Experimental de Silvicultura Tropical - INPA - AM, durante o período de outubro de 1998 a março de 1999.

\section{DISCUSSÃO}

Na região amazônica brasileira, estão registradas cerca de 157 espécies do gênero Lutzomyia, no qual estão incluídas as principais espécies transmissoras de Leishmanioses na região (Young \& Duncan, 1994). Assim, das 41 espécies de flebótomos coletadas na nossa área de estudos, 29 delas (71\%) picam o homem (com maior ou menor intensidade), principalmente dos subgêneros Nyssomyia e Psychodopygus, como demonstra o trabalho de Young \& Arias (1992).

Quando comparamos a fauna de flebótomos da área de estudos do presente trabalho, com o trabalho realizado por Arias \& Freitas (1982a), também em uma área preservada de floresta primária de terra firme da Reserva Florestal Ducke - INPA, utilizando armadilhas luminosas CDC em diferentes alturas, encontramos 27 espécies em comum: L.amazonensis, L. anduzei, L. aragaoi, L. ayrozai, L. dendrophyla, L. dreisbachi, L. flaviscutellata, L. furcata, L. inpai, L. lutziana, L. migonei, L. monstruosa, L. olmeca nociva, L. pacae, L. paraensis, L. pilosa, L. rorotaensis, $L$. ruii, $L$. sericea, $L$. shannoni, $L$. squamiventris squamiventris, $L$. triacantha, $L$. trichopyga, L. trispinosa, L. tuberculata e $L$. umbratilis.

O Gênero Brumptomyia com 0,02\%, juntamente com as espécies do Grupo Aragaoi, foram pouco representativas em nossas amostras, pois são específicas de buracos de tatus, e necessitam de tipos de armadilhas específicas para suas coletas (Young \& Arias, 1992). Resultados semelhantes foram obtidos por Arias \& Freitas (1982 a) quando coletaram apenas 3 indivíduos de $B$. pintoi.

Outras espécies como L. inpai, $L$. lutziana, L. pacae, L. pilosa, L. triacantha, L. trispinosa e L. bispinosa, foram coletadas com pouca frequência, semelhante ao trabalho de Arias \& Freitas (1982 a), que utilizaram os mesmos métodos de captura.

As espécies $L$. flaviscutellata e L olmeca nociva, não foram coletadas na área de estudos, com tanta eficácia quanto as espécies $L$. umbratilis e $L$. anduzei, por não ser o método de captura (armadilhas CDC) o mais utilizado para as referidas espécies, que é através da armadilha Disney.

Arias et al. (1987) demonstraram a importância da espécie L. olmeca nociva, juntamente com L. flaviscutellata, como vetoras de L. amazonensis, demonstrando também a presença de casos humanos de leishmaniose causados por L. amazonensis na região de Manaus e na Rodovia BR 174. Apesar de existir uma capacidade de transmitir o parasito, $L$. olmeca nociva, juntamente com L. reducta, possuem um pequeno papel na ecologia e 
epidemiologia (Lainson \& Shaw, 1999).

Ready et al. (1983) citaram que $L$. infraspinosa e $L$. pacae são fortemente rodentofílicos; $L$. choti, L. trichopyga e $L$. aragaoi são comuns em buracos de tatus. $L$. anduzei, L. umbratilis, L. davisi, L. paraensis, L. ayrozai e L. squamiventris são antropófilas e L. flaviscutellata é fortemente antropófíla.

As espécies L. ayrozai e L. davisi, recentemente foram envolvidas na transmissão de Leishmania naiffi Lainson \& Shaw, 1989, agente responsável pela leishmaniose cutânea, na região do Amazonas e Pará (Lainson \& Shaw, 1989).

No Equador, em área endêmica na floresta primária e secundária, a mais importante espécie antropofílica $L$. gomezi tem sido considerada um suposto vetor de leishmaniose. Segundo Mendonza-León et al. (1997) as espécies Leishmania ( Viannia ) colombiensis Kreutzer, Corredor, Grimaldi, Grol, Rowton, Young, Morales, McMahonPratt, Guzman \& Tesh, 1991 e $L$. ( $V$ ) panamensis Lainson \& Shaw, 1978, são veiculadas por L. gomezi. Ainda, segundo Christensen et al. (1983) no Panamá e Young \& Rogers (1984) no Equador, a L. gomezi pode também transmitir a L. braziliensis.

O principal vetor dentre os animais silvestres para os humanos é a espécie $L$. umbratilis, com infecções relativamente pouco frequentes encontradas em L. anduzei (Lainson \& Shaw, 1999).

Barrett (1993) demonstrou que $L$. umbratilis é normalmente a espécie dominante em amostras de flebótomos coletados com armadilhas de luz na floresta primária de terra firme, na região de Manaus, ao norte do Rio Amazonas, onde representou 36\% dos flebótomos coletados em Balbina, Amazonas, a aproximadamente $60 \mathrm{Km}$ da área do presente estudo. Além disso, conforme os nossos resultados, o subgênero Nyssomyia (68\%) foi predominante nas coletas em Balbina, seguido do subgênero Psychodopygus (18,28\%).

Em capturas feitas durante 63 semanas no interior de uma floresta primária, Arias \& Freitas (1982b) obtiveram 50 diferentes espécies entre 21.026 indivíduos coletados, das quais, aproximadamente $70 \%$ das espécies coletadas foram L. umbratilis e L. anduzei.

Recentemente no Norte do Estado do Mato Grosso, estudos mostraram a presença de importantes vetores como $L$. umbratilis, $L$. whitmani e L. flaviscutellata. A espécie $L$. umbratilis ocorreu em alta densidade, foi a mais antropofílica e foi encontrada naturalmente infectada por parasitos localizados na secção Peripilaria, caracterizados como L. braziliensis (Rangel et al., 1999).

A espécie L. umbratilis é o maior transmissor da L. guyanensis ao norte do sistema fluvial do Rio Negro (Arias \& Freitas, 1978). Ao sul desse sistema, $L$. anduzei e $L$. umbratilis foram capturados, todavia ainda não foram encontradas infectadas com leishmanias, mas sim atacando o homem.

Os flebótomos do subgênero Psathyromyia, L. dendrophyla, L. scaffi e $L$. shannoni foram pouco atraídos por armadilhas de luz em Balbina (Barrrett, 1993), o que está de acordo com os resultados do presente trabalho. Estas espécies, principalmente $L$. shannoni, tem sido associadas com a transmissão de Endotrypanum schaudinni Mesnil \& Brimont 1908, um tripanosomatídeo exclusivo de preguiças (Arias et al., 1985).

A espécie L. rorotaensis, a quinta a aparecer com maior frequência em nossas coletas, tem sido encontrada ocasionalmente infectada com tripanosomatídeos, provavelmente de répteis, como a lagartixa Thecadactylus rapicaudus (Lainson \& Shaw, 1979). Estudos usando técnicas moleculares (KDNA) para a detecção de parasitos em flebótomos também demonstraram infecção por Endotrypanum em L. shannoni, L. umbratilis e L. anduzei (Franco \& Grimaldi, 1999).

No Pará, L. tuberculata foi coletada com uma espécie desconhecida de leishmania, assinalada no subgênero Viannia (KillickKendrick, 1990). Ainda, segundo este autor, 16 tripanosomatídeos foram isolados de $L$. umbratilis, 1 de L. ayrozai e 13 de $L$. squamiventris squamiventris, porém não foram identificados. A larga proporção de casos de leishmaniose cutânea no Brasil é devido a L.braziliensis e L. guyanensis, ambas com vetores que se alimentam no homem; ao contrário das infecções humanas causadas por L. lainsoni e $L$. naiffi relativamente raras, com seus vetores não atraídos para picar pessoas (Lainson \& Shaw, 1999).

Uma oitava espécie, a $L$. deanei estaria também presente na Amazônia, porém até o momento ainda não foi identificada como sendo transmissora da doença na população humana (Paes et al., 1998).

Outros flebótomos coletados e que são prováveis ou suspeitos vetores de leishmanias, 
foram os seguintes (Killick-Kendrick, 1990): $L$. furcata (Leishmania deanei Lainson \& Shaw, 1977); L. amazonensis, L. ayrozai, L. paraensis e L. migonei (Leishmania braziliensis); L. tuberculata (Leishmania guyanensis); L. gomezi (Leishmania panamensis); L. ayrozai e $L$. paraensis (Leishmania naiffi).

A semelhança no número total de espécies coletadas, com $14 \%$ de $L$. anduzei (em relação a $19 \%$ de L. umbratilis), na abundância sazonal durante os meses de coleta, além do encontro de um dos exemplares infectados por flagelados, nos leva a argumentar sobre a real importância da espécie no ciclo ecoepidemiológico da doença. A espécie $L$. anduzei, além das semelhanças morfológicas externas, parece ter um comportamento semelhante a $L$. umbratilis.

As diferenças entre machos e fêmeas coletadas com as armadilhas $\mathrm{CDC}$, como ocorrido nos resultados deste trabalho, podem ser atribuídas entre outras causas, à necessidade maior de dispersão das fêmeas do que os machos para o repasto sanguíneo e a oviposição (Tabela 1). Chaniotis et al. (1971) citou alguns fatores que poderiam corroborar às diferenças na captura de machos e fêmeas, tais como fêmeas de certas espécies serem mais facilmente atraídas do que machos, diferenças na taxa de mortalidade, sítios de repouso e controle genético.

A composição florística das florestas tropicais influenciam na composição faunística dos flebótomos, seja pela produção de efeitos distintos no solo da floresta (Rutledge \& Ellenwood, 1975) ou pelas características peculiares dos troncos das árvores (Geoffroy et al., 1986; Cabanillas \& Castellón, 1999). As larvas dos flebótomos, que se alimentam de matéria orgânica em decomposição, incluindo pequenos organismos do solo (Hanson, 1968), certamente são afetadas por mudanças na fauna edáfica e alterações microclimáticas.

O comportamento das diferentes espécies de flebótomos varia de acordo com a estratificação vertical, ou seja, em relação as diferentes alturas e ao nível do solo. Esse dados obtidos no presente estudo, foram descritos no trabalho de Dias-Lima et al. (2002).

Os padrões eco-epidemiológicos das doenças tem sido influenciados pela perda de florestas naturais tropicais causadas pelo desmatamento, favorecendo por exemplo adaptabilidade de algumas espécies como $L$. flaviscutellata e Proechimys $s p$. à plantações exóticas e o não estabelecimento de $L$. umbratilis, que preferencialmente sugam Edentados em florestas primárias de terra firme e que não se adaptam as copas das vegetações exóticas (Walsh et al., 1993; Ready et al., 1983). Segundo Pessoa (2000) a fauna de flebótomos dendrobata e suas taxas de infecção natural por tripanosomatídeos, diminui sensivelmente, após a extração seletiva de madeira, sendo uma forte evidência de que este grupo de insetos sofre impacto direto na sua população, quando ocorre a extração.

A LTA apresenta uma evidente variação sazonal, sendo a transmissão da doença mais intensa no período mais chuvoso, quando a temperatura, insolação, evaporação estão mais baixas e a umidade alta, parecem ser mais propícias ao aumento da densidade dos flebótomos. Chuvas prolongadas têm ação mecânica direta, afetando as populações dos insetos, como por exemplo os flebótomos, que se recolhem aos seus sítios de repouso para proteção. $\mathrm{Na}$ estação seca, diminui a precipitação e a umidade relativa do ar e do solo. Quando as folhas caídas no solo estavam totalmente secas e com o aumento da temperatura, houve pouquíssima captura de flebótomos. Esse fato pode explicar o insucesso na captura com a armadilhas luminosas nos meses de agosto e setembro (período de précoleta), fato também mostrado nos trabalhos de Arias \& Freitas (1977) e Andrade (1998). A estação seca parece ser, por motivos biológicos intrínsecos desse grupo de insetos, um período redução no nível populacional dos flebótomos. Uma outra hipótese é que os ovos dos flebótomos, postos em criadouros selecionados pelas fêmeas, ficam aguardando um momento propício, com um limite mínimo de umidade, para que haja eclosão das larvas.

Outros fatores como temperatura mínima noturna, chuvas, vento, fase lunar, altura de vôo e atraente sexual (feromônios), podem influenciar na abundância e atração dos insetos pela luz. Dentre esses fatores, pudemos observar que houve uma menor captura dos flebótomos nas noites em que ocorreram chuvas prolongadas, geralmente acompanhadas de ventos e neblina. Todos esses fatores abióticos devem ser melhor estudados para o melhor conhecimento das flutuações das populações dos flebótomos. 
Tabela 1 - Total, porcentagem e relação entre sexos dos flebotomíneos coletados com armadilhas luminosas CDC, na Estação Experimental de Silvicultura Tropical - INPA, durante o período de outubro de 1998 à março de 1999.

\begin{tabular}{|c|c|c|c|c|c|}
\hline ESPÉCIES & TOTAL & $\%$ & M & $\mathrm{F}$ & Razão sexual - M:F \\
\hline 1. gênero Brumptomyia & 2 & 0,02 & 1 & 1 & \\
\hline B. pintoi Costa lima, 1932 & 1 & 0,01 & 1 & 0 & $1: 0$ \\
\hline B. $\mathrm{sp}$ & 1 & 0,01 & 0 & 1 & $0: 1$ \\
\hline 2. gênero Lutzomyia & 7407 & 9,98 & 2694 & 4711 & \\
\hline sub-gênero Evandromyia Mangabeira, 1941 & 482 & 6,49 & 31 & 451 & \\
\hline L. infraspinosa Mangabeira, 1941 & 396 & 5,34 & 9 & 387 & $1: 43$ \\
\hline L. inpai Young \& Arias, 1977 & 5 & 0,06 & 0 & 5 & $0: 1$ \\
\hline L. monstruosa Floch \& Abonnenc, 1944 & 81 & 1,09 & 22 & 59 & $1: 2,7$ \\
\hline grupo Aragaoi Theodor, 1965 & 47 & 0,63 & 15 & 32 & \\
\hline L. aragaoi Costa Lima, 1932 & 46 & 0,62 & 14 & 32 & $1: 2,3$ \\
\hline L. inflata Floch \& Abonnenc, 1944 & 1 & 0,01 & 1 & 0 & $1: 0$ \\
\hline grupo Dreisbachi Theodor, 1965 & 89 & 1,20 & 58 & 31 & \\
\hline L. dreisbachi Causey \& Damasceno, 1945 & 89 & 1,20 & 58 & 31 & $1: 1,9$ \\
\hline grupo Migonei Theodor, 1965 & 132 & 1,77 & 66 & 66 & \\
\hline L. migonei França, 1920 & 30 & 0,40 & 11 & 19 & $1: 1,7$ \\
\hline L. pacae Floch \& Abbonenc, 1943 & 3 & 0,04 & 2 & 1 & $1: 0,5$ \\
\hline L. sericea Floch \& Abbonenc, 1944 & 99 & 1,33 & 53 & 46 & $1,1: 1$ \\
\hline grupo Oswaldoi Theodor, 1965 & 545 & 7,35 & 67 & 478 & \\
\hline L. rorotaensis Floch \& Abbonenc, 1944 & 545 & 7,35 & 67 & 478 & $1: 7,1$ \\
\hline grupo Pilosa Theodor, 1965 & 2 & 0,02 & 0 & 2 & \\
\hline L. pilosa Damasceno \& Causey, 1944 & 2 & 0,02 & 0 & 2 & $0: 2$ \\
\hline grupo Verrucarum Theodor, 1965 & 2 & 0,02 & 2 & 1 & \\
\hline L. sp. & 2 & 0,02 & 2 & 1 & $1: 0,5$ \\
\hline sub-gênero Lutzomyia França, 1924 & 84 & 1,13 & 16 & 68 & \\
\hline L. gomezi Nitzulescu, 1931 & 84 & 1,13 & 16 & 68 & $1: 4,2$ \\
\hline sub-gênero Mycropygomyia, Barretto, 1962 & 1 & 0,01 & 1 & 0 & \\
\hline L. cayennensis ? Floch \& Abonnenc, 1941 & 1 & 0,01 & 1 & 0 & $1: 0$ \\
\hline sub-gênero Nyssomyia Barreto, 1962 & 2923 & 39,43 & 1123 & 1800 & \\
\hline L. anduzei Rozeboom, 1942 & 1051 & 14,18 & 500 & 551 & $1: 1,1$ \\
\hline L. flaviscutellata Mangabeira, 1942 & 102 & 1,37 & 40 & 62 & $1: 1,5$ \\
\hline L. olmeca nociva Young \& Arias, 1982 & 375 & 5,06 & 132 & 243 & $1: 1,8$ \\
\hline L. umbratilis Ward \& Fraiha, 1977 & 1395 & 18,82 & 451 & 944 & $1: 2,1$ \\
\hline sub-gênero Pressatia Mangabeira, 1942 & 74 & 0,99 & 19 & 55 & \\
\hline L. choti Floch \& Abonnenc, 1941 & 47 & 0,63 & 7 & 40 & $1: 5,7$ \\
\hline L. trispinosa Mangabeira, 1942 & 27 & 0,36 & 12 & 15 & $1: 1,2$ \\
\hline sub-gênero Psathyromyia Barretto, 1962 & 122 & 1,63 & 51 & 71 & \\
\hline L. dendrophyla Mangabeira, 1942 & 49 & 0,66 & 13 & 36 & $1: 2,6$ \\
\hline L. Iutziana Costa Lima, 1932 & 20 & 0,26 & 11 & 9 & $1,2: 1$ \\
\hline L. shannoni Dyar, 1929 & 53 & 0,71 & 27 & 26 & $1: 1$ \\
\hline sub-gênero Psychodopygus Mangabeira, 1941 & 1681 & 22,68 & 505 & 11,72 & \\
\hline L. amazonensis Root, 1934 & 346 & 4,66 & 96 & 245 & $1: 2,5$ \\
\hline L. ayrozai Barretto \& coutinho, 1942 & 181 & 2,44 & 64 & 118 & $1: 1,8$ \\
\hline L. bispinosa Fairchild \& Hertig, 1951 & 2 & 0,02 & 2 & 0 & $1: 0$ \\
\hline L. davisi Root, 1934 & 568 & 7,66 & 161 & 407 & $1: 2,5$ \\
\hline L. geniculata Mangabeira, 1941 & 104 & 1,44 & 4 & 100 & $1: 25$ \\
\hline L. paraensis Costa Lima, 1941 & 183 & 2,46 & 69 & 114 & $1: 1,6$ \\
\hline L. squamiventris squamiventris Lutz \& Neiva, 1912 & 297 & 4,00 & 109 & 188 & $1: 1,7$ \\
\hline sub-gênero Sciopemyia Barretto, 1962 & 98 & 1,31 & 36 & 63 & \\
\hline L. nematoducta Young \& Arias, 1984 & 82 & 1,10 & 25 & 57 & $1: 2,3$ \\
\hline L. pennyi Arias \& Freitas, 1981 & 16 & 0,21 & 11 & 5 & $2,2: 1$ \\
\hline sub-gênero Trichophoromyia Barretto, 1962 & 24 & 0,32 & 3 & 21 & \\
\hline L. ruii Arias \& Young, 1982 & 24 & 0,32 & 3 & 21 & $1: 7$ \\
\hline sub-gênero Trichopygomyia Barretto, 1962 & 793 & 10,69 & 642 & 151 & \\
\hline L. raticliffei Arias, Ready \& Freitas & 17 & 0,22 & 17 & 0 & $1: 0$ \\
\hline L. trichopyga Floch \& Abonnenc, 1945 & 776 & 10,47 & 625 & 151 & $4,2: 1$ \\
\hline sub-gênero Viannamyia Mangabeira, 1941 & 138 & 1,85 & 40 & 98 & \\
\hline L. furcata Mangabeira, 1941 & 82 & 1,10 & 39 & 43 & $1: 1,1$ \\
\hline L. tuberculata mangabeira, 1941 & 56 & 0,75 & 1 & 55 & $1: 55$ \\
\hline (flebótomos danificados não identificados) & 171 & 2,30 & 19 & 152 & \\
\hline INDIVÍDUOS & 7409 & $100 \%$ & 2695 & 4712 & $1: 1,7$ \\
\hline
\end{tabular}




\section{BIBLIOGRAFIA CITADA}

Andrade, S.L. 1998. Leishmaniose tegumentar Americana em área de ocupação recente na periferia da cidade de Manaus, Estado do Amazonas, Brasil. Dissertação de Mestrado Instituto Oswaldo Cruz / FIOCRUZ, Rio de Janeiro, 206p.

Arias, J.R.; Freitas, R.A. 1977. Flebótomos da Amazônia Central do Brasil. I. Resultados obtidos das capturas com isca humana e equina (Diptera: Psychodidae). Acta Amazonica, 7 (4): 507-527.

Arias, J.R.; Freitas, R.A. 1978. Sobre os vetores de leishmaniose cutânea na Amazônia Central do Brasil. 2: incidência de flagelados em flebótomos selváticos. Acta Amazonica, 8(3): 387-396.

Arias, J.R.; Freitas, R.A. 1982a. On the vectors of cutaneous leishmaniasis in the Central Amazon of Brazil. 3. Phlebotomine sand fly stratification in a terra firme forest. Acta Amazonica, 12(3): 599-603.

Arias, J.R.; Freitas, R.A. 1982b. On the vectors of cutaneous leishmaniasis in the Central Amazon of Brazil. 4. Sand fly emergence from a "terra firme forest floor. Acta Amazonica, 12 (3): 609-611.

Arias, J.R.; Freitas, R. A; Naiffi, R.D.; Barrett, T.V. 1987. Observations on the parasite Leishmania mexicana amazonensis and its natural infection of the sand fly Lutzomyia olmeca nociva. P.A.H. O Bulletin 21 (1): 48-53.

Arias, J.R.; Miles, M. A; Naiffi, R.D.; Popova, M. M.; Freitas, R.A; Biancardi, C.B.; Castellón, E.G. 1985. Flagellates infections of brazilian sandflies (Díptera: Psychodidae): isolation in vitro and biochemical identification of Endotrypamum e Leishmania. Amer. J. Trop. Med. Hyg., 34: 10981108.

Barrett, T.V. 1993. Cutaneous leishmaniasis in Amazonas State, Brazil: eco-epidemiology and questions of control. Proceedings of National Workshop Research and Control of Leishmaniasis in Brazil, Recife, 31-34.

Barrett, T.V.; Freitas, R.A. Naiff, M.F.; Naiff, R.D. 1991. As leishmanias e seus transmissores em relação a saúde na
Amazônia. In: Val, A.L.; Figliuolo, R.; Feldberg, E. (Eds). Bases Cientificas para Estratégias de Preservação e Desenvolvimento da Amazônia: Fatos e perspectivas. Vol. 1, Instituto Nacional de Pesquisas da Amazônia, Manaus, 105117.

Barrett, T.V.; Freitas, R.A. Albuquerque, M.I.C.; Guerrero, J.C.H. 1996. Report on a colection of Lutzomyia sand flies (Diptera : Psychodidae) from the Middle Solimões (Amazonas, Brazil). Mem. Inst. Oswaldo Cruz, 9(1): 27-35.

Cabanillas M.R.S.; Castellón, E.G. 1999. Distribution of sandflies (Diptera: Psychodidae) on tree-trunks in a non-flooded area of the Ducke Forest Reserve, Mamaus, AM, Brazil. Mem. Inst. Oswaldo Cruz, 94(3): 289-296.

Chaniotis, B.N.; Correa, M.A.; Tesh, R.B.; Jonhson, K.M. 1971. Daily and seasonal man-biting activity of Phlebotomine sandflies in Panama. J. Med. Ent., 8 (4): 415-420.

Christensen, H.A.; Fairchild, G.B.; Herrer, A.; Johnson, C.M.; Young, D.G.; Vasquez, A.M. 1983. The ecology of cutaneous leishmaniasis in eastern Panamá. II. Entomological investigations. Ann. Trop. Med. Parasitol., 66:55-66.

Dias-Lima, A.G.; Castellón, E.C.; Medeiros, J.F. \& Sherlock I.A. 2002. Estratificação vertical da fauna de flebotomíneos (Díptera: Psychodidae) de uma floresta primária de terra firme da Amazônia central, Amazonas, Brasil. Cad. Saúde Publica, 18 (3): 823-823.

Franco, A.M.R.; Grimaldi, G. 1999. Characterization of Endotrypanum (Kinetoplastida: Trypanosomatidae), a unique parasite infecting the neotropical tree sloths (Edentata). Mem. Inst. Oswaldo Cruz, 94 (2): 261-268.

Geoffroy, B.; Dedet, J.P.; Lebbe, J.; Esterre, P.; Trape, J.F. 1986. Note sur les relations des vecteurs de leishmaniose avec les essences forestieres en Guyane Française. Ann. Parasitol. Hum. Comp., 61 (4): 491-505. 
Guerra, J.A.O.; Onety, A.C.; Santos, S.L.; Santos, F.G.C.; Talhari, S.; Paes, M.G. 2001. Situação da Leishmaniose em Manaus na última década. Rev. Soc. Bras. Med. Trop., 34 (suppl 1): 244.

Hanson, W.S. 1968. The immature stages of the subfamily Phlebotominae in Panama (Diptera: Psychodidae). Tese de Doutorado, Universidade de Kansas, EUA. 206p.

Killick-Kendrick, R. 1990. Phlebotomine vectors of the leishmaniases: a rewiew. Med. Vet. Entomol., 4: 1-24.

Lainson, R. 1997. On Leishmania enriettii and other enigmatic Leishmania species of the neotropics. Mem. Inst. Oswaldo Cruz, 92 (3): $377-387$.

Lainson R; Shaw J.J. 1979. The role of animals in the epidemiology of South American Leishmaniasis, WHR Lumsden; D.A. Evans Edit. Biology of Kinetoplastida, Academic Press, vol 2, 738p.

Lainson R; Shaw J.J. 1989. Leishmania (Viannia) naiffi sp.n. a parasite of the armadillo, Dasypus novencinctus (L.) in Amazonian Brazil. Ann. Parasitol. Hum. Comp., 64 (1):3-9.

Lainson R; Shaw J.J. 1999. New World Leishmaniases - the Neotropical Leishmania species. In Cox, F.E.G.; Kreier, J.P.; Wakelin, D. Eds. "Topley \& Wilson's microbiology and microbial infections parasitology" London, Sidney, Aucklond: 241266.

Lainson, R.; Shaw, J.J., Ward, R.D.; Ready, P.D.; Naiff, R.D. 1979. Leishmaniasis in Brazil: XIII. Isolation of Leishmania from armadillos (Dasypus novencictus) and observations on the epidemiology of cutaneous leishmaniasis in North Para State. Trans. Roy. Soc. Trop., Med. Hyg. 73: 239242.

Mendoza-León A.; Shaw, J.J.;Tapia, F.J. 1997. A guide for cutaneous leishmaniasis connoiseur. In Tapia, F.J.; CáceresDittmar, G.; Sanchez, M.A. Eds. "Molecular and immune mechanisms in the phatogenesis of cutaneous leishmaniasis" R.G. Londes Company, Austin, Texas, 1-14.
Paes, M.G.; Barros, M.L.B.; Toledo, L.M. 1998. Considerações sobre a produção da Leishmaniose Tegumentar Americana no estado do Amazonas. In. Rojas, L.I. Espaço e doenças : um olhar sobre o Amazonas, Rio de Janeiro, Fiocruz, 175 p. il.

Pessoa, F.A.C. 2000. Efeitos da extração de madeira sobre flebotomineos (Diptera: Psychodidae) em uma floresta de produção: abundância relativa das espécies em troncos de árvores e prevalência de tripanosomatídeos. Dissertação de Mestrado Instituto Nacional de Pesquisas da Amazônia / Universidade do Amazonas, Manaus, xiv $+87 \mathrm{p}$.

Pimenta, P.F.P.; Saraiva, E.M.B.; Rowton, E.; Modi, G.B.; Garraway, L.A.; Beverley, S.M.; Turco, S.J.; Sacks, D.L. 1994. Evidence that the vectorial competence of phlebotomine sand flies for different species of Leishmania is controlled by structural polymorphisms in the surface lipophosphoglycan. Proc. Natl. Acad. Sci. USA, 91 : 9155-9159.

Rangel, E.F.; Azevedo, A C. R.; Lima, J. B.; Souza, N.A; Pereira, T.; Meneses, C.R.V.; Costa, V.A. 1999. Ecologia da leishmaniose cutânea no estado do Mato Grosso. I. Distribuição vertical da fauna flebotomínica (Díptera: Psychodidae: Phlebotominae). Rev. Soc. Bras. Med. Trop., 32 (Suppl. I), $25 \mathrm{p}$.

Ready, P.D.; Lainson, R., Shaw, J.J. 1983. Leishmaniasis in Brazil: XX. Prevalence of ënzootic rodent leishmaniasis" (Leishmania mexicana amazonensis) and apparent absence of "pian bois"(Leishmania braziliensis guyanensis) in plantations of introduced tree species and others non-climax forests in eastern Amazônia. Trans. Roy. Soc. Trop. Med. Hyg. 77 (6): 775-785.

Rutledge, L.C.; Ellenwood, D.A. 1975. Production of phlebotomine sandflies on the open forest floor in Panama: Phytologic and Edaphic relations. Environmental Entomology, 4(1):83-89.

Sherlock, I.A.; Maia, H; Dias-Lima, A.G. 1996. Resultados preliminares de um projeto 
sobre a ecologia dos flebótomos vetores de leishmaniose tegumentar no Estado da Bahia. Rev. Soc. Bras. Med. Trop., 29(2): 207214.

Walsh, J.F.; Molyneux, D.H.; Birley, M.H. 1993. Deforestation: effects on vectorborne disease. Parasitology, 106:555-575.

Young, D.G.; Rogers T.E. 1984. The phlebotomine sand fly fauna (Diptera: Psychodidae) of Equador. J. Med. Entomol., 21: 597-611.

Young, D.G.; Arias, J.R. 1992. Flebótomos vectores de Leishmaniasis en las Americas. OPAS, cuaderno tecnico, $\mathrm{n}^{\circ} 33,28 \mathrm{p}$.

Young, D. G.; Duncan, M.A. 1994. Guide to identification and geographic distribution of Lutzomyia sandflies in Mexico, West Indies, Central and South America (Diptera: Psychodidae). Associated Publishers, American Entomological Institute, $881 \mathrm{p}$. 